\title{
Clinical assessment tools identify functional deficits in fragility fracture patients
}

This article was published in the following Dove Press journal:

Clinical Interventions in Aging

5 May 2016

Number of times this article has been viewed

\section{Tyler D Ames' \\ Corinne E Wee' \\ Khoi M Le' \\ Tiffany L Wang' \\ Julie Y Bishop ${ }^{2}$ \\ Laura S Phieffer ${ }^{2}$ \\ Carmen E Quatman²}

'The Ohio State University College of Medicine, ${ }^{2}$ Department of Orthopaedics, The Ohio State University Wexner Medical Center, Columbus, $\mathrm{OH}$, USA
Correspondence: Carmen E Quatman Department of Orthopaedics, The Ohio State University Wexner Medical Center, 410 W I0th Avenue, Columbus, $\mathrm{OH}$ 43210 , USA

Tel +l 6142938652

Email carmen.quatman@osumc.edu
Purpose: To identify inexpensive, noninvasive, portable, clinical assessment tools that can be used to assess functional performance measures that may put older patients at risk for falls such as balance, handgrip strength, and lumbopelvic control.

Patients and methods: Twenty fragility fracture patients and 21 healthy control subjects were evaluated using clinical assessment tools (Nintendo Wii Balance Board [WBB], a handheld dynamometer, and an application for the Apple iPod Touch, the Level Belt) that measure functional performance during activity of daily living tasks. The main outcome measurements were balance (WBB), handgrip strength (handheld dynamometer), and lumbopelvic control (iPod Touch Level Belt), which were compared between fragility fracture patients and healthy controls.

Results: Fragility fracture patients had lower scores on the vertical component of the WBB Torso Twist task $(P=0.042)$ and greater medial-lateral lumbopelvic sway during a $40 \mathrm{~m}$ walk $(P=0.026)$ when compared to healthy controls. Unexpectedly, the fracture patients had significantly higher scores on the left leg $(P=0.020)$ and total components $(P=0.010)$ of the WBB Single Leg Stand task as well as less faults during the left Single Leg Stand task $(P=0.003)$.

Conclusion: The clinical assessment tools utilized in this study are relatively inexpensive and portable tools of performance measures capable of detecting differences in postural sway between fragility fracture patients and controls.

Keywords: fall risk, geriatric fracture, Nintendo Wii Balance Board, Level Belt, fragility fracture

\section{Introduction}

Fall-related injuries in the elderly population are a rapidly growing public health concern that lead to a wide range of financial, physical, and emotional complications. One out of three elderly adults fall each year in the US, resulting in more than 1.5 million fragility fractures annually. ${ }^{1,2}$ Due to the aging baby boomer generation, these numbers will continue to grow. The impact of these injuries will also continue to be an economic burden, with the cost for fall-related injuries projected to exceed US $\$ 30$ billion by $2020 .^{3}$ Fragility fractures often leave patients in great emotional distress and unable to perform activities of daily living. ${ }^{4,5}$ The impact of these fractures is dramatic enough that a survey of older women indicated that $80 \%$ of them preferred death to a hip fracture that would result in admission to a nursing home. ${ }^{5}$ Mortality for patients with fragility fractures is also significantly increased compared to expected mortality rates for this demographic population. ${ }^{6}$ A multicenter Canadian study found that hip fracture patients had a 4.2 times greater risk of death during the first year after fracture than would be expected for hip fracture patients. ${ }^{7}$ Mortality 5 years after hip or vertebral fracture is also $\sim 20 \%$ greater than would be expected for elderly individuals without fracture. ${ }^{4}$ 
Identifying functional deficits that may put elderly patients at risk for falls is crucial to help prevent the morbidity and mortality that can occur after a fragility fracture. Impaired balance, lumbopelvic control, and handgrip strength all have shown strong correlations with fall risk. ${ }^{8-13}$ Rehabilitation studies demonstrate that both improvements in postural sway and increases in strength measures are possible in geriatric patients after a fragility fracture. ${ }^{14-16}$ In addition, multiple systematic reviews have shown that targeted interventions, mainly exercise and balance exercises, can reduce fall risk in elderly patients, thus preventing future complications. ${ }^{17-20}$ Because of the high morbidity and mortality associated with falls in the elderly, the American and British Geriatric Societies recommend that all the elderly individuals who present for medical attention because of a fall, report recurrent falls in the past year, or report difficulties in walking or balance should have a multifactorial fall risk assessment performed to determine their future fall risk..$^{21}$ This assessment should include a detailed evaluation of gait, balance, and lower extremity joint function.

Given the great morbidity and mortality complications with fall-related injuries in the elderly, along with the potential for functional improvement after treatment and rehabilitation, there is a need to develop inexpensive, objective, and portable technologies to help evaluate people for fall risk quickly and accurately. The Affordable Health Care Act emphasizes improvement of quality and efficiency of health care, public health, access to innovative medical therapies, and prevention of chronic disease. As a result, the orthopedic surgery field faces new socioeconomic pressures to develop performance-based criteria and evidence-based clinical assessment tools to evaluate outcomes of treatment for musculoskeletal injuries. Three clinical assessment tools were identified and utilized to assess stability (Wii Balance Board [WBB], iPod Touch Level Belt), postural sway (Wii Balance Board, iPod Touch Level Belt), and handgrip strength (Saehan handheld dynamometer).

Inclusion criteria required that each tool be commercially available, easily portable, reasonably inexpensive, and operational with little training or expertise. The WBB (Nintendo, Redmond, WA, USA) was selected to evaluate a patient's balance and stability. Recent studies have already demonstrated that the WBB has the ability to detect subtle changes in balance and center of pressure with good test-retest reliability when compared to the gold standard, a researchgrade force platform. ${ }^{22,23}$ The iPod Touch Level Belt (Perfect Practice, Columbus, OH, USA) was selected to evaluate a patient's lumbopelvic control. The iPod Touch Level Belt is an iPod Touch application available in the Apple iTunes store and has been used to measure postural sway in baseball players during functional tasks. ${ }^{24}$ The built-in accelerometer of the iPod Touch (Apple, Cupertino, CA, USA) has already been shown to reliably and validly assess gait and posture in the elderly population. ${ }^{25}$ Accelerometry has been used in other studies to measure trunk stability in the elderly population and has been suggested as a possible screening tool for future fall risk. ${ }^{26}$ The handheld dynamometer was selected to evaluate a patient's handgrip strength. Handheld dynamometers have been used to measure handgrip in many studies to provide quick and accurate strength quantification., ${ }^{9,16,27-29}$ In addition, decreased isometric handgrip strength has been identified as a risk factor for future falls in a 1-year prospective study. ${ }^{10}$ The goal of this study was to identify inexpensive, noninvasive, portable tools that can be used to assess potential deficits - including balance, handgrip strength, and lumbopelvic control - that may put elderly patients at risk for falls. We hypothesize that our tools will be able to quickly and easily identify balance, strength, and stability deficits in fragility fracture patients in a variety of clinical settings.

\section{Patients and methods Subjects}

Prior to study participation, all subjects signed informed consent and agreed to participate in the study as approved by the The Ohio State University's Biomedical Sciences Institutional Review Board. The participants were recruited via fliers from three orthopedic outpatient clinics and the general community. The two groups evaluated were patients with a history of one or more fragility fractures and controls without a history of fragility fractures. A fragility fracture was defined as a fracture occurring from a fall from a standing height or less, without major trauma such as a motor vehicle accident. The fracture must have occurred after the patient was 50 years of age. ${ }^{30}$ Further inclusion criteria required that all subjects in the study be 50 years old or greater, able to walk 40 feet unassisted, able to follow simple verbal and written directions, and have an absence of peripheral neuropathies or mobility disorders.

Each participant completed a written questionnaire about age, sex, weight, current ambulatory status, fragility fracture details, and activity limitations. Included in the patient questionnaire was a section from the 36-Item Short Form Health Survey (SF-36), which evaluates daily activity restrictions based on the subject's current state of health. Participants were then asked to perform a series of functional assessment 
activities over the course of 20 minutes utilizing the clinical assessment tools in a prognostic case-control study.

\section{Procedure}

\section{Stability}

Stability was evaluated using the Nintendo Wii video game platform and the WBB. In conjunction with the WBB, two "mini-games" (Torso Twists and Single Leg Stand) from the Wii Fitness program were used to assess postural sway and stability in participants. All hardware and software is commercially available and was not altered from its purchased state. Participants were asked to remove their shoes and stand on the WBB facing a member of the research team. For each trial, the researcher navigated the on-screen menu and selected the activity to be performed. In addition, the researcher also performed the activity at the same time as the participant to ensure that the correct movements and pace were maintained throughout the duration of the exercise. As shown in Figure 1, the researcher was able to see the Wii instructions on the TV screen, but the screen was blocked from the participant's view.

The first activity selected from the Wii Fitness program was the Torso Twist activity, which requires patients to raise their arms parallel to the ground and rotate it $90^{\circ}$ to one side,

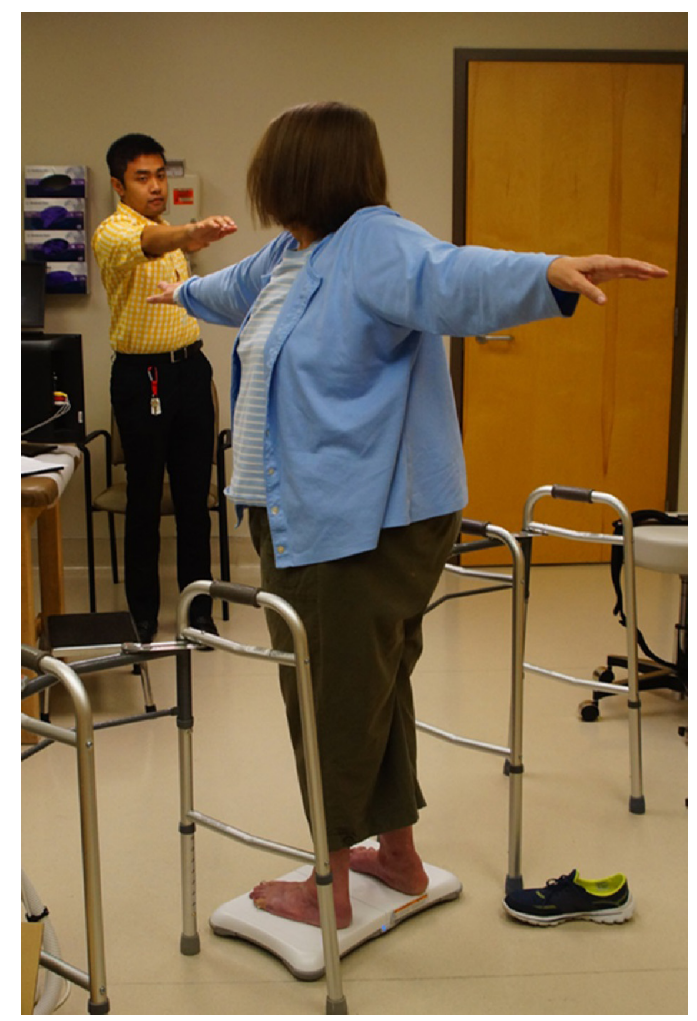

Figure I Setup of researcher, participant, TV, and Wii Balance Board during the Torso Twist balance task. then to the other, alternating sides for a total of three twists to each side. At the end of the trial, horizontal, vertical, and total balance scores were reported. The maximum horizontal and vertical scores were 50 for each, and the maximum total score was 100 , which was a sum of the horizontal and vertical components. The vertical score correlated to the amount of shift in the anterior-posterior (AP) direction, while the horizontal score correlated to shift in the medial-lateral (ML) direction. Higher scores represented decreased postural sway throughout the duration of the task. This activity was repeated three times per patient.

The second activity selected from the Wii Fitness program was the Single Leg Stand activity which measures balance as the participant performs a series of movements while standing on one leg. The recommended motion from the Wii Fitness program was simplified by only asking patients to "stand with your hands on your hips and raise your leg off the ground". Participants were told that if they felt unstable, they could briefly grab the safety bars that were placed on either side of them or tap their raised foot down, but to reset to the one-leg stand position as soon as possible. The trial lasted 15 seconds on each leg. At the end of each trial, right, left, and total balance scores were reported. Again, the maximum right and left scores were 50 for each, and the maximum total score was 100 , which was a sum of the right and left components. As with the Torso Twist activity, higher scores indicated decreased postural sway throughout the duration of the task. In addition, hand and foot faults were measured as a secondary internal measure of balance and sway. This activity was repeated three times per patient.

\section{Handgrip strength}

Handgrip strength was evaluated using the Saehan Squeeze Handheld Dynamometer. Participants were asked to hold their forearm parallel to the ground with their elbow flexed at $90^{\circ}$ and pointed laterally. Participants were asked to "grip as hard as they can for 3 seconds, then release". Encouragement was provided to each participant throughout the task and they were told when to release. This procedure was repeated three times on each hand.

\section{Lumbopelvic control}

Lumbopelvic control was evaluated using the iPod Touch Level Belt application. The apparatus consists of an elastic belt to which an iPod Touch is attached by Velcro (Velcro USA, Manchester, NH, USA). The belt is placed across both of the participant's posterior superior iliac spines with the iPod Touch centered in the middle of the back. 


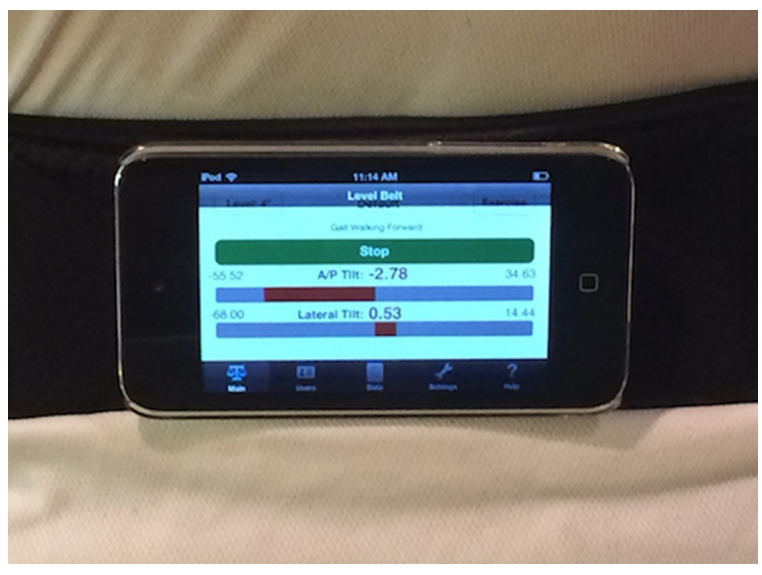

Figure 2 Placement and screen display of iPod Touch Level Belt measuring lumbopelvic tilt during a $40 \mathrm{~m}$ walk.

The application uses an accelerometer-based sensor to measure AP and ML tilt as the participant walks. The participant is instructed to walk normally for $40 \mathrm{~m}$ to the end of the hall and stop. The screen displayed during the walking trial is shown in Figure 2. The data obtained during the middle half of the walking stage were used in the study in order to capture the normal gait phase of walking and not acceleration or deceleration phases of a walk. The procedure was repeated three times.

\section{Statistical analysis}

Torso Twist and Single Leg Stand balance scores reported by the WBB as well as faults during the Single Leg Stand were averaged over the three trials. For handgrip strength, the best score out of three trials for each hand was used for statistical analysis. The iPod Touch Level Belt records the AP and ML tilt in degrees during the walking trials 60 times per second. For both the AP and ML tilt data, the standard deviation of the tilt data during the middle half of the walking stage was calculated. Two-sample Student's $t$-tests were conducted to compare balance, handgrip strength, and lumbopelvic tilt between fragility fracture patients and controls. Normality assumptions of the $t$-tests were investigated, and sensitivity analyses using nonparametric methods were conducted to confirm the results. Chi-square test was performed to compare sex difference between the groups.

\section{Results}

The participants comprised 20 older adults with a history of fragility fracture and 21 older adults with no history of fragility fracture. Subject demographics are summarized in Table 1. The distribution of fragility fracture sites in the fracture group is summarized in Table 2. No significant differences were
Table I Summary of participant demographics

\begin{tabular}{llll}
\hline Subjects & Fracture & Control & $P$-value \\
\hline $\mathrm{N}$ & 20 & $2 \mathrm{I}$ & \\
$\mathrm{Sex}$ & & & \\
$\quad$ Female & $14(70.0 \%)$ & $1 \mathrm{I}(52.4 \%)$ & 0.248 \\
$\quad$ Male & $6(30.0 \%)$ & $10(47.6 \%)$ & \\
Age (years) & $64.0 \pm 8.8$ & $62.4 \pm 8.9$ & 0.573 \\
Weight (kg) & $77.8 \pm 18.8$ & $86.5 \pm 17.2$ & 0.136 \\
Activity level (SF-36) & $2.11 \pm 0.90$ & $2.66 \pm 0.42$ & $0.026 *$ \\
\hline
\end{tabular}

Notes: $* P<0.05$ was considered statistically significant. Mean \pm SD is reported for the continuous data.

Abbreviations: SF-36, 36-Item Short Form Health Survey; SD, standard deviation.

observed in sex, age, or weight when comparing fracture patients to control patients. The participants' average activity level was significantly lower in the fracture group when compared to the control group ( $P=0.026)$. Results from the two-sample $t$-tests showed that participants with a history of fragility fracture had lower scores on the vertical component of the WBB Torso Twist Task $(P=0.042)$ and increased ML sway during a $40 \mathrm{~m}$ walk $(P=0.026)$ when compared to controls. Fracture patients also had higher left leg and total balance scores as well as less faults on the left leg than control patients during the Single Leg Stand task on the WBB ( $P=0.020,0.010,0.003$, respectively). There was no statistical difference in horizontal or total balance score during the WBB Torso Twist, right leg scores during the Single Leg Stand task, right leg or total faults during the Single Leg Stand task, dominant or nondominant handgrip strength, or AP sway during a $40 \mathrm{~m}$ walk when comparing the two groups. Results of all clinical assessment tool testing are displayed in Table 3. Comparisons of WBB scores and iPod Touch Level Belt results between the fracture patients and controls can be seen in Figures 3 and 4, respectively.

\section{Discussion}

The purpose of this study was to identify inexpensive, noninvasive, portable tools that can be used to assess potential deficits that may put elderly patients at risk for falls. Current techniques used to measure gait and balance often require

Table 2 Distribution of fragility fracture sites in the experimental group

\begin{tabular}{lllll}
\hline Side & $\begin{array}{l}\text { Upper } \\
\text { extremity }\end{array}$ & $\begin{array}{l}\text { Lower } \\
\text { extremity }\end{array}$ & $\begin{array}{l}\text { Both upper and } \\
\text { lower extremities }\end{array}$ & Total \\
\hline Right-sided & 5 & 6 & 1 & 12 \\
Left-sided & 3 & 3 & 0 & 6 \\
Bilateral & 0 & 0 & 2 & 2 \\
Total & 8 & 9 & 3 & 20 \\
\hline
\end{tabular}

Note: Location of fragility fracture sites in the experimental group was determined by a chart review conducted in June 2014 . 
Table 3 Results of clinical assessment tools testing comparing fracture patients with controls

\begin{tabular}{llll}
\hline Test & Fracture & Control & P-value \\
\hline $\mathrm{N}$ & 20 & 21 & \\
TT horizontal avg & $33.8 \pm 7.2$ & $32.7 \pm 7.8$ & 0.660 \\
TT vertical avg & $44.3 \pm 4.8$ & $46.9 \pm 3.7$ & $0.042^{*}$ \\
TT total avg & $77.6 \pm 9.9$ & $79.7 \pm 8.9$ & 0.482 \\
SLS left avg & $42.8 \pm 4.3$ & $35.9 \pm 0.0$ & $0.020^{*}$ \\
SLS right avg & $37.6 \pm 6.7$ & $30.7 \pm 13.3$ & 0.085 \\
SLS total avg & $80.4 \pm 9.6$ & $66.6 \pm 20.0$ & $0.010^{*}$ \\
SLS left faults avg & $0.17 \pm 0.28$ & $1.02 \pm 1.12$ & $0.003^{*}$ \\
SLS right faults avg & $0.50 \pm 0.48$ & $0.82 \pm 0.96$ & 0.208 \\
SLS total faults avg & $0.93 \pm 1.06$ & $1.83 \pm 1.94$ & 0.086 \\
Max D grip strength & $20.9 \pm 8.1$ & $23.9 \pm 10.5$ & 0.334 \\
Max ND grip strength & $20.5 \pm 6.8$ & $23.5 \pm 9.4$ & 0.268 \\
Avg AP SD & $1.71 \pm 0.47$ & $1.63 \pm 0.42$ & 0.563 \\
Avg ML SD & $1.91 \pm 0.42$ & $1.58 \pm 0.46$ & $0.026^{*}$ \\
\hline
\end{tabular}

Notes: $* P<0.05$ was considered statistically significant. Mean \pm SD is reported for the continuous data. Scores reported by the Wii Balance Board were averaged over three trials. Avg AP and ML SD refers to the average standard deviation of the anterior-posterior and medial-lateral tilt in degrees as measured by the iPod Touch level belt during the middle $50 \%$ of a $40 \mathrm{~m}$ walk.

Abbreviations: $\mathrm{D}$, dominant; ND, nondominant; SD, standard deviation; TT, Torso Twist activity on the Wii Balance Board; SLS, Single Leg Stand activity; avg, average; Max, maximum; AP, anterior-posterior; ML, medial-lateral.

highly specialized, expensive, and cumbersome equipment. These factors make it difficult to identify balance deficits in elderly patients conveniently and efficiently. ${ }^{28}$ The Torso Twist evaluation on the WBB is able to assess a patient's real-time balance during a reaching exercise, very similar to situations that occur when actual falls take place. In addition, the iPod Touch Level Belt is able to measure real-time sway during normal walking. Handgrip dynamometry uses a simulation of gripping handrails and has been shown to be highly predictive of functional limitations and disability later in life. ${ }^{28,31}$ These clinical assessment tools are able to assess patients in situations very closely mimicking normal daily activities, thus allowing the results from this study to be highly clinically relevant. The portability and accessibility of these tools in a clinical orthopedic setting allow for patient data collection in convenient ways that a biomechanics laboratory cannot provide. In addition, commonly used balance evaluations, such as the Berg Balance Scale and the Timed Up and Go Test, do exist; however, these evaluation tools are limited in their ability to detect subtle changes and often cannot distinguish between fallers and nonfallers..$^{22,29,32}$ In contrast, the clinical assessment tools used in this study were able to detect differences in postural sway between fragility fracture patients and controls.

All of the clinical assessment tools were easily transported into nearly any small-sized exam room and could be set up within 10 minutes. This study took place at multiple locations and demonstrates the potential to use this technology as part of multi center studies. The total cost of all equipment used was under US $\$ 1,000$. All tools were commercially available and unmodified from their factory state, allowing any clinical site to easily adopt and utilize our testing protocol. The entire evaluation for one patient was consistently completed in less than 20 minutes. A previous pilot study conducted with nine advanced practice providers demonstrated that all nine participants could be trained in under an hour to effectively and confidently use the clinical assessment tools to evaluate patients for fall risk in a clinical setting. ${ }^{33}$ This study demonstrates that these clinical assessment tools identified are portable, affordable, and efficient to use in clinical settings.

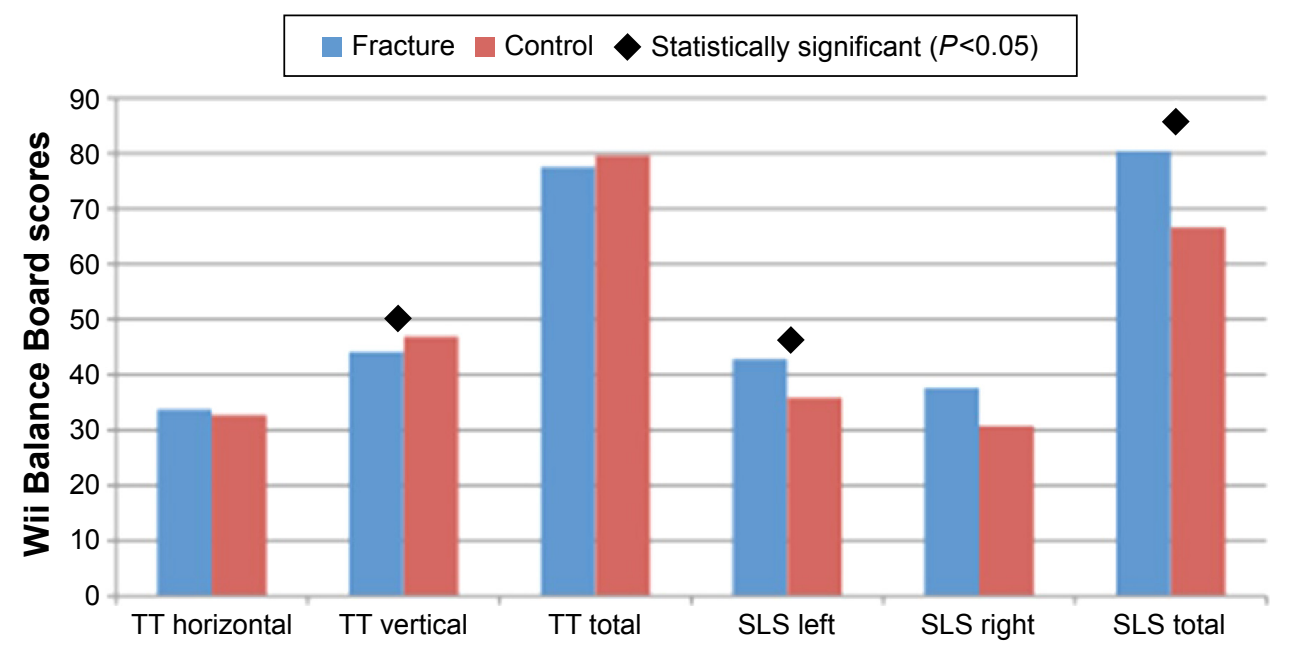

Figure 3 Comparison of Wii Balance Board scores in fracture patients and controls. Abbreviations: TT, Torso Twist activity on the Wii Balance Board; SLS, Single Leg Stand activity. 


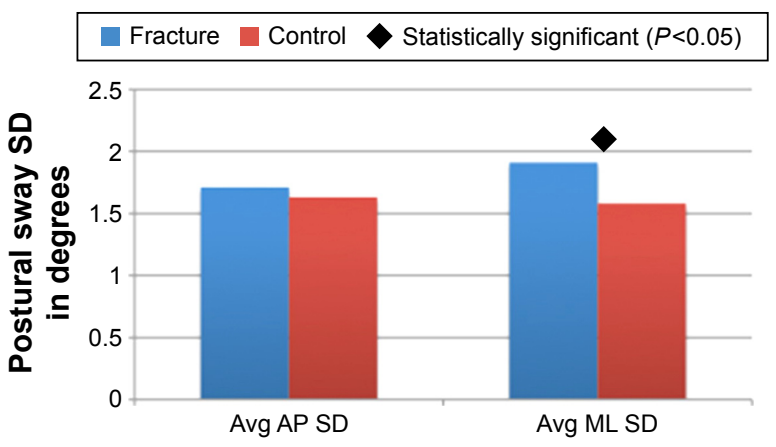

Figure 4 Comparison of iPod Level Belt postural sway in fracture patients and controls. Note: Avg AP and ML SD refers to the average standard deviation of the anteriorposterior and medial-lateral tilt in degrees as measured by the iPod Touch level. Abbreviations: Avg, average; AP, anterior-posterior; ML, medial-lateral; SD, standard deviation.

After sustaining an initial fragility fracture, patients are 2-3 times more likely to sustain a second future fracture than the general population. ${ }^{34,35}$ Thus, being proactive in regard to evaluating patients for fall risk and initiating fall prevention measures at earlier ages is crucial in this high-risk population. Clinical assessment tools provide opportunities for both fall risk assessment and patient-specific rehabilitation. For example, these tools can be used to identify a patient's specific weaknesses in stability and strength, from which an individualized rehabilitation plan can be developed to address each patient's specific functional needs. Clinical assessment tools can continue to be involved in the rehabilitation process, as well in the assessment of a patient's recovery process.

In our analysis of participant demographics, the daily activity level of the fracture patients was significantly decreased when compared to controls. Given that fragility fractures have been shown to limit patients' ability to complete activities of daily living, this finding seemed logical., Coinciding with our initial hypothesis, the clinical assessment tools were able to detect both increased ML sway and lower scores on the WBB Torso Twist task in fracture patients when compared to controls. While dominant and nondominant handgrip strength for fracture patients were approximately $3 \mathrm{~kg}$ lower than the controls, the differences were not statistically significant $(P=0.334,0.268)$.

Unexpectedly, the fracture patients had significantly higher scores on the left leg and total components of the WBB Single Leg Stand task than the controls, indicating decreased postural sway. The right leg component for the fracture patients, although not significant, was still higher than the scores for the controls $(P=0.085)$. Paralleling these findings, the fracture patients recorded significantly less faults during the left leg task and less total faults when compared to the controls $(P=0.003,0.085)$. One possible explanation for this difference is that the fracture patients concentrate harder on staying balanced because they are more concerned about falling than the control patients. Healthy individuals may also naturally have a more random pattern of sway as a strategy to adapt to changes in the environment, and individuals with injuries lose this compensatory mechanism and demonstrate decreased sway during balance tasks. A similar phenomenon was also reported in a study measuring postural coordination in athletes who have sustained an anterior cruciate ligament injury. ${ }^{36}$ Interestingly, when looking at a more functional dynamic task of gait compared to single leg balance, these same postural sway deficits actually reversed and fragility fracture patients had decreased postural sway control (increased ML sway). Gait requires a different level of concentration with a controlled perturbation in ground contact with each step. This is compelling evidence that variability of movement during different tasks may reveal functional deficits in fragility fracture patients that may put them at risk for future fracture and falls.

\section{Study limitations}

The fragility fracture population has the potential for baseline balance strength and stability deficits that put them at risk for future falls. This study revealed that this population differs from healthy control subjects in functions measured by the clinical assessment tools, and these tools identified some deficits associated with a high risk for falling. While the risk factors identified in this pathologic population were not their baseline measures associated with falling risk, these tests are promising to be used in future longitudinal study to predict the risk for future falls. Additional information about the subjects such as socioeconomic status, prefracture activity levels, and motivation levels could improve risk stratification in our fragility fracture group. However, one of the main goals of this study was to identify tools that could assess fragility fracture patients in a clinical setting both quickly and efficiently.

In addition, portable technology inherently has some limitations, such as the possible need for calibration with each use and the simplification of internal equipment. Both the Wii and handheld dynamometer tests are well validated in the literature. ${ }^{10,14,23,28,37}$ The iPod Touch Level Belt has also been validated; however, it is relatively a newer technology and more validation studies are emerging each year related to the technology. ${ }^{24}$ The analysis for the iPod Touch Level Belt was performed by the techniques detailed by the inventors, and this emerging technology is being used in high level sport and rehabilitation settings. ${ }^{24}$ Similar accelerometer-based testing using the iPod Touch has demonstrated high validity 
and reliability during gait and posture testing in adults of all ages. ${ }^{25}$ The differences in handgrip strength may not have reached significance due to the smaller sample size, and future work will investigate this further.

\section{Conclusion}

The clinical assessment tools utilized in this study are relatively cheap, portable, and were able to detect differences in postural sway between fragility fracture patients and controls. These tools can provide real-time, objective functional data inexpensively, efficiently, and in nearly any clinical setting. In the orthopedic trauma setting, fractures in the elderly population lead to profound morbidity, mortality, and social and financial consequences for the patients and the orthopedic community. Utilization of clinical assessment tools to identify patients at risk for falls and future fractures may help clinicians develop targeted intervention strategies to help prevent future falls and fragility fractures. Prevention of even a small percentage of fragility fractures could have substantial medical and economic benefits for society.

\section{Acknowledgments}

The authors thank the staff of The Ohio State University Hand and Upper Extremity Center, Martha Morehouse, and Carepoint East for their help in the completion of this study. This work was supported in part by The Ohio State University College of Medicine Roessler Research Scholarship (TDA).

\section{Disclosure}

The authors report no conflicts of interest in this work.

\section{References}

1. Chang JT, Morton SC, Rubenstein LZ, et al. Interventions for the prevention of falls in older adults: systematic review and meta-analysis of randomised clinical trials. BMJ. 2004;328(7441):680.

2. US Department of Health and Human Services. Bone Health and Osteoporosis: A Report of the Surgeon General. Rockville, MD: US Department of Health and Human Services, Office of the Surgeon General; 2004.

3. Englander F, Hodson TJ, Terregrossa RA. Economic dimensions of slip and fall injuries. J Forensic Sci. 1996;41(5):733-746.

4. Cooper $\mathrm{C}$. The crippling consequences of fractures and their impact on quality of life. Am J Med. 1997;103(2A):12S-17S; discussion 17S-19S.

5. Salkeld G, Cameron ID, Cumming RG, et al. Quality of life related to fear of falling and hip fracture in older women: a time trade off study. BMJ. 2000;320(7231):341-346.

6. Magaziner J, Simonsick EM, Kashner TM, Hebel JR, Kenzora JE. Survival experience of aged hip fracture patients. Am J Public Health. 1989; 79(3):274-278.

7. Ioannidis G, Papaioannou A, Hopman WM, et al. Relation between fractures and mortality: results from the Canadian Multicentre Osteoporosis Study. CMAJ. 2009;181(5):265-271.

8. National Osteoporosis Foundation. Physician's Guide to the Prevention and Treatment of Osteoporosis. Washington, DC: National Osteoporosis Foundation; 2003.
9. Chang SH, Mercer VS, Giuliani CA, Sloane PD. Relationship between hip abductor rate of force development and mediolateral stability in older adults. Arch Phys Med Rehabil. 2005;86(9):1843-1850.

10. Lloyd BD, Williamson DA, Singh NA, et al. Recurrent and injurious falls in the year following hip fracture: a prospective study of incidence and risk factors from the Sarcopenia and Hip Fracture study. J Gerontol A Biol Sci Med Sci. 2009;64(5):599-609.

11. Ganz DA, Bao Y, Shekelle PG, Rubenstein LZ. Will my patient fall? JAMA. 2007;297(1):77-86.

12. Maki BE, Holliday PJ, Topper AK. A prospective study of postural balance and risk of falling in an ambulatory and independent elderly population. J Gerontol. 1994;49(2):M72-M84.

13. Pluijm SM, Smit JH, Tromp EA, et al. A risk profile for identifying community-dwelling elderly with a high risk of recurrent falling: results of a 3-year prospective study. Osteoporos Int. 2006;17(3):417-425.

14. Clark R, Kraemer T. Clinical use of Nintendo Wii bowling simulation to decrease fall risk in an elderly resident of a nursing home: a case report. J Geriatr Phys Ther. 2009;32(4):174-180.

15. Sylliaas H, Brovold T, Wyller TB, Bergland A. Prolonged strength training in older patients after hip fracture: a randomised controlled trial. Age Ageing. 2012;41(2):206-212.

16. Mangione KK, Craik RL, Palombaro KM, Tomlinson SS, Hofmann MT. Home-based leg-strengthening exercise improves function 1 year after hip fracture: a randomized controlled study. J Am Geriatr Soc. 2010; 58(10):1911-1917.

17. Gillespie LD, Robertson MC, Gillespie WJ, et al. Interventions for preventing falls in older people living in the community. Cochrane Database Syst Rev. 2012;9(9):CD007146.

18. Province MA, Hadley EC, Hornbrook MC, et al. The effects of exercise on falls in elderly patients. A preplanned meta-analysis of the FICSIT Trials. Frailty and Injuries: Cooperative Studies of Intervention Techniques. JAMA. 1995;273(17):1341-1347.

19. Sherrington C, Whitney JC, Lord SR, Herbert RD, Cumming RG, Close JC. Effective exercise for the prevention of falls: a systematic review and meta-analysis. J Am Geriatr Soc. 2008;56(12):2234-2243.

20. El-Khoury F, Cassou B, Charles MA, Dargent-Molina P. The effect of fall prevention exercise programmes on fall induced injuries in community dwelling older adults: systematic review and meta-analysis of randomised controlled trials. BMJ. 2013;347(347):f6234.

21. The American Geriatrics Society. Clinical Practice Guideline: Prevention of falls in older person; 2010. Available from: http://www.americangeriatrics. org/health_care_professionals/clinical_practice/clinical_guidelines_ recommendations/2010/. Accessed February 12, 2016.

22. Clark RA, Bryant AL, Pua Y, McCrory P, Bennell K, Hunt M. Validity and reliability of the Nintendo Wii Balance Board for assessment of standing balance. Gait Posture. 2010;31(3):307-310.

23. Koslucher F, Wade MG, Nelson B, Lim K, Chen FC, Stoffregen TA. Nintendo Wii Balance Board is sensitive to effects of visual tasks on standing sway in healthy elderly adults. Gait Posture. 2012;36(3):605-608.

24. Chaudhari AM, McKenzie CS, Borchers JR, Best TM. Lumbopelvic control and pitching performance of professional baseball pitchers. J Strength Cond Res. 2011;25(8):2127-2132.

25. Kosse NM, Caljouw S, Vervoort D, Vuillerme N, Lamoth CJ. Validity and reliability of gait and postural control analysis using the tri-axial accelerometer of the iPod Touch. Ann Biomed Eng. 2015;43(8):1935-1946.

26. Lamoth CJ, van Deudekom FJ, van Campen JP, Appels BA, de Vries OJ, Pijnappels M. Gait stability and variability measures show effects of impaired cognition and dual tasking in frail people. J Neuroeng Rehabil. 2011;8(2):2.

27. Thorborg K, Bandholm T, Holmich P. Hip- and knee-strength assessments using a hand-held dynamometer with external belt-fixation are inter-tester reliable. Knee Surg Sports Traumatol Arthrosc. 2013;21(3): 550-555.

28. Samuel D, Rowe P, Hood V, Nicol A. The relationships between muscle strength, biomechanical functional moments and health-related quality of life in non-elite older adults. Age Ageing. 2012;41(2):224-230.

29. Blum L, Korner-Bitensky N. Usefulness of the Berg Balance Scale in stroke rehabilitation: a systematic review. Phys Ther. 2008;88(5):559-566. 
30. Bawa HS, Weick J, Dirschl DR. Anti-osteoporotic therapy after fragility fracture lowers rate of subsequent fracture: analysis of a large population sample. J Bone Joint Surg Am. 2015;97(19):1555-1562.

31. Rantanen T, Masaki K, He Q, Ross GW, Willcox BJ, White L. Midlife muscle strength and human longevity up to age 100 years: a 44-year prospective study among a decedent cohort. Age (Dordr). 2012;34(3): 563-570.

32. Schoene D, Wu SM, Mikolaizak AS, et al. Discriminative ability and predictive validity of the timed up and go test in identifying older people who fall: systematic review and meta-analysis. J Am Geriatr Soc. 2013; 61(2):202-208.

33. Wang TL, Quatman CE, Ames TD, et al. Training advanced practice providers to collect functional outcomes after fragility fractures. Geriatr Orthop Surg Rehabil. 2014;6(3):130-139.
34. Klotzbuecher CM, Ross PD, Landsman PB, Abbott TA 3rd, Berger M. Patients with prior fractures have an increased risk of future fractures: a summary of the literature and statistical synthesis.JBone Miner Res. 2000; 15(4):721-739.

35. van Staa TP, Leufkens HG, Cooper C. Does a fracture at one site predict later fractures at other sites? A British cohort study. Osteoporos Int. 2002; 13(8):624-629.

36. Kiefer AW, Ford KR, Paterno MV, et al. Inter-segmental postural coordination measures differentiate athletes with ACL reconstruction from uninjured athletes. Gait Posture. 2013;37(2):149-153.

37. Ruff J, Wang TL, Quatman-Yates CC, Phieffer LS, Quatman CE. Commercially available gaming systems as clinical assessment tools to improve value in the orthopaedic setting: a systematic review. Injury. 2015;46(2): $178-183$.
Clinical Interventions in Aging

\section{Publish your work in this journal}

Clinical Interventions in Aging is an international, peer-reviewed journal focusing on evidence-based reports on the value or lack thereof of treatments intended to prevent or delay the onset of maladaptive correlates of aging in human beings. This journal is indexed on PubMed Central, MedLine,

\section{Dovepress}

CAS, Scopus and the Elsevier Bibliographic databases. The manuscript management system is completely online and includes a very quick and fair peer-review system, which is all easy to use. Visit http://www.dovepress. com/testimonials.php to read real quotes from published authors. 